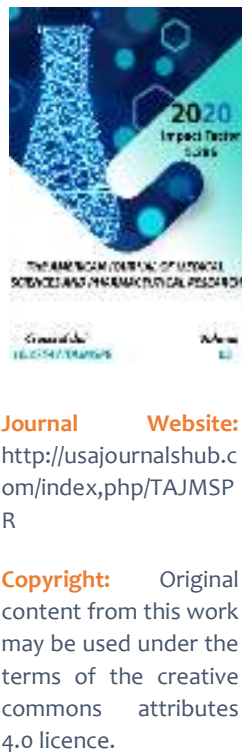

\title{
Job Of Serum Heart Troponin-I For Chance Separation In First Assault In Intense Myocardial Dead Tissue
}

\author{
Mohammed Belal \\ Department Of Biochemistry, Sylhet Women's Medical College, Bangladesh \\ Shafiul Alam \\ Department Of Biochemistry, Sylhet Women's Medical College, Bangladesh
}

\section{ABSTRACT}

Cardiovascular troponin-I (cTnl) is known to have the most noteworthy explicitness and logical affectability for recognition of myocardial injury; it is utilized both as indicative and prognostic marker. This examination was expected to affirm this thought. Subjects and techniques: This imminent observational investigation included 60 patients of 40 to 65 years age go analyzed as intense myocardial dead tissue. The mean ages were $50 \pm 8$ years and $53 \pm 8$ years in Q - wave AMI and non Qwave AMI separately. Male and female patients included were $86.7 \%$ and $13.3 \%$; BMI was $25.3 \pm 1.5$. Results: Study demonstrated troponin-I $7.53 \pm 0.086 \mathrm{ng} / \mathrm{ml}$ in Q wave and in non Q-wave AMI was $6.38 \pm 0.64 \mathrm{ng} / \mathrm{ml}$ following 24 hours of assault of AMI with no critical distinction between two gatherings ( $P>0.05)$. The mean troponin-I inside 9 hours of assault, were $1.60 \pm 0.80 \mathrm{ng} / \mathrm{ml}$ and $2.7 \pm 1.4$ $\mathrm{ng} / \mathrm{ml}$ in steady and unsteady gathering individually and the distinction discovered measurably huge $(P<0.05)$

\section{KEYWORDS}

Cardiovascular Troponin-I, intense myocardial localized necrosis, hazard delineation.

\section{INTRODUCTION}

Ischaemic coronary illness is a significant worldwide medical issue and the most widely recognized reason for untimely bleakness and mortality among men in the created world1. Death rates credited to IHD differ between 
nations. The WHO MONICA study observing IHD and hazard factors in 38 thousand populace from 21 nations in four landmasses demonstrated that age normalized yearly occasion rates in 1985 to 1987 in men extend from 915/100,000 in North korelia, Finland to $76 / 100,000$ in Beijing, China. records three rules for the determination of AMI; chest torment, electrocardigaphic changes and increments in Biochemical markers4. Absence of demonstrative affectability and particularity of clinical and traditional markers forestall or postpone the treatment, prompting the excessive enduring to the patients. As of not long ago, the regular initial phase in diagnosing AMI patients whose manifestations are good with ischaemia is a cautious history and physical assessment.

\section{MATERIALS AND TECHNIQUES}

This forthcoming observational contextual analysis included 60 patients age extend 40multi year analyzed as dynamic myocardial localized necrosis by ECG and heart markers inside 24 hours ofattack and conceded in CCU of Zainul Haque Sikder cardiovascular consideration and examination enter, Dhaka. Study was directed in the branch of organic chemistry, BSMMU during January 2002 to December 2003. Moral endorsement was taken from the authority of the concerned medical clinic and educated assent was taken from all members. Three back to back blood tests were gathered from every one of the investigation subjects. Initial one inside 9 hours of assault, second example between 9-24 hours of assault and third one following 24 hours of assault however inside the day of confirmation were taken and continue. All tests were performed upon the arrival of assortment. All patients were followed up upto 30 days of assault by taking history; clinical assessment, Biochemical examination and imaging method.

\section{RESULT}

Absolute 60 patients were remembered for this investigation of which 30 were $Q$ wave $A M I$ and 30 were non $\mathrm{Q}$ wave AMI. The male patients remembered for this examination were $86.7 \%$ and female were $13.3 \%$ with the male; female proportion 6.5:1. The diverse standard attributes of study subjects in regard of Q-wave AMI and non Q-wave AMI which didn't contrast. The mean troponin-I in Q-wave AMI inside 9 hours, between 9-24 hours and

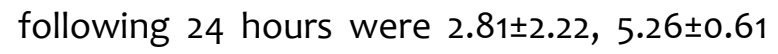
and $7.53 \pm 0.86 \mathrm{ng} / \mathrm{ml}$ and those in non Q-wave AMI were $2.35 \pm 0.39,3.93 \pm 0.58$ and $6.38 \pm 0.64$ $\mathrm{ng} / \mathrm{ml}$ separately with no noteworthy contrast between two gatherings ( $P>0.05)$.

\section{CONVERSATION}

In this forthcoming observational investigation we have assessed the serum cardiovascular troponin-I for chance delineation in post myocardial dead tissue of patients first at any point assaulted by MI. All out 60 patients with an ongoing history of chest torment analyzed as Q-wave AMI (30) and non Q-wave AMI (30) by regular E. C. $G$ and heart markers inside 24 hours of assault and conceded in CCU were chosen for the investigation.

\section{DISCUSSION}

Conversation In this planned observational investigation we have assessed the serum heart troponin-I for hazard delineation in post 
myocardial dead tissue of patients first ever assaulted by MI. Absolute 60 patients with an ongoing history of chest torment analyzed as Q-wave AMI (30) and non Q-wave AMI (30) by customary E. C. $G$ and heart markers inside 24 hours of assault and conceded in CCU were chosen for the examination.

Despite the fact that Q-wave AMI professed to have more myocardial harm than non Q-wave AMI. Our discoveries of serum cTnl neglected to

uphold this since the centralization of these cardiovascular markers was not diverse between two gatherings. This was additionally enhanced by our discoveries of no contrasts between two gatherings concerning this unfavorable cardiovascular result. This may be because of the way that the myocardial harm in Q-wave AMI were sufficiently only to make Q-wave in ECG yet not yet to cause rushed arrival of heart markers. Test frameworks for heart troponin-I (cTnl) give the most noteworthy particularity and logical affectability for recognition of myocardial injury and serum cTnl assists with evaluating the genuine complexity of the patients11. A few different investigations likewise have reported the prognostic prevalence of the serum cTnl for right on time and safe danger separation of patients with intense chest pain12.

The examination led by Bodi expressed that troponin-I is better as an indicative tool13. Braunwald suggested that troponin-I as the best heart markers for symptomatic and prognostic reason. The employments of cardiovascular troponin will undoubtly expands the quantity of occasion recorded specifically preliminary in view of increment affectability for distinguishing Ml14.

\section{CONCLUSION}

Heart troponin-I is the most explicit and delicate marker of myocardial cell injury and in this way have supplanted creatinine kinase MB. Heart troponin-I has high particularity for cardiovascular injury since it isn't found in skeletal muscle during neonatal turn of events and adulthood. Heart troponin-I is discharged into blood inside hours of the beginning of side effects of myocardial dead tissue and that it stays raised for a few days of post-localized necrosis.

\section{REFERENCES}

1. Antman EM, Guess in ischaemic heart infections. Medication Worldwide 1995; 4(2):184-188.

2. Steinberg WJ, Balfe DI, The study of disease transmission of ischaemic coronary illness. Medication Global 1995; 4(12):419-433.

3. Milenko JR Kannel WB, Review examination of intense myocardial localized necrosis. Blast Heart J 1989; 11:140-145.

4. Braunwald $\mathrm{M}$, Goldman L.Similar affectability of cardiovascular troponin I and lactate dehydrogenase isoenzymes for diagnosing intense MI. Clin Chem 2004; 12:270-276. 
5. Bodi VV. Heart troponin-1: A marker with high explicitness for cardiovascular injury. Flow 1995; 38: 401-406. 